$\frac{12}{12} / 25-9585(1)$

UCRL-ID-118533

\title{
Pencil Beam Radar Selectivity of Ocean Wave Spectra
}

\author{
R. Manasse
}

August 1, 1994

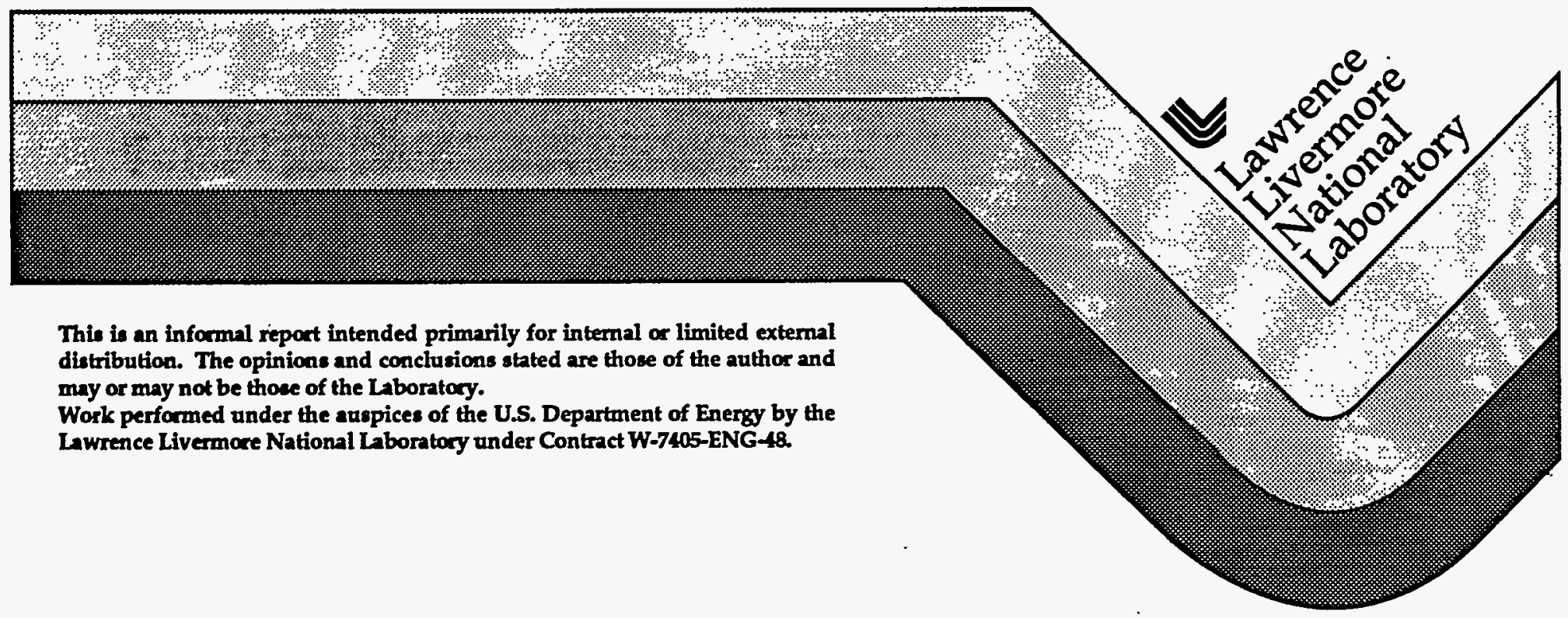

$d s$ 


\section{DISCLAIMER}

This document was prepared as an account of work sponsoned by an agency of the United States Government. Neither the United States Government nor the University of California nor any of their employees, makes any warranty, express or implied, or assumes any legal liability or responsibility for the accuracy, completeness, or usefulness of any information, apparatus, product, or process disclosed, or represents that its use would not infringe privately owned rights. Reference herein to any specific commercial product, process, or service by trade name, trademark, menufacturer, or otherwise, does not necessarily constitute or imply its endorsement, recommendation, or favoring by the United States Government or the University of Californin. The views and opinions of authors expressed herein do not necessarily state or reflect those of the United States Government or the University of California, and shall not be used for advertising or product endorsement purposes.

This report has been reproduced directly from the best available copy.

Available to DOE and DOE contractors from the Office of Scientific and Technical Information P.O. Box 62, Oak Ridge, IN 37831

Prices available from (615) 576-B401, FIS 626-8401

Available to the public from the National Technical Information Service

U.S. Deportment of Commerce

5285 Port Royal Rd. Springfield, VA 22161 


\section{DISCLAIMER}

Portions of this document may be illegible in electronic image products. Images are produced from the best available original document. 


\section{Introduction and Summary}

Over the past several years some very interesting data relevant to ocean wave backscatter have been produced by non scanning pencil beam radars with high range resolution. These radars are located on land which over looks the water/sea surface and operate at low grazing angles (LGA). Prominent among these are the $X$-band RSRE-Thorn EMI and the LLNL-Hughes real aperture radars employed in the Joint US/UK ocean wave experiments in Loch Linnhe and the sound of sleat (scotland). The range-time image in Fig. 1 [1] obtained with the RSRE radar is typical of the many images which have been obtained. The most prominent features of these images are linear regions or strips of increased average RCS having temporal durations up to or exceeding 100 seconds. For HH polarization these features are marked by the presence of periodic spikes in RCS. The features appear quite consistent with wave groups which are moving approximately parallel to the radar boresight. A self-consistent model of this wave group behavior, as manifested in the radar images, has been proposed by Tulin [2]. This model provides estimates of the wavelengths involved and accounts for the periodicity of the spiky returns which appear to be due to the passage of nonlinear or incipiently breaking waves through the wave groups. Nothing in this model precludes the presence of multiple wave groups moving at different velocities. In fact, some images exhibit wave groups moving at different velocities, and in some cases both incoming and outgoing wavegroups are present simultaneously [3].

Obviously, $X$-band Bragg waves cannot explain these features because the lifetime of these waves is, at best, a few seconds. The association of these features with the scattering from the crests of much larger (e.g., meters) waves is reasonable because the lifetime of these waves is very long. However, it is not reasonable to assume that ambient wavegroups in the field of view of the radar all just happen to be moving along the radar boresight. One would expect that the totality of waves in the ambient sea background moving in various directions would produce a fairly rapid temporal decorrelation of the features seen in these images, but this view is clearly contradicted by the data. One explanation for this apparent contradiction is that this type of radar is highly selective in its response to wave (and thus wave group) direction. In other words, the radar acts as a spatial filter which passes waves moving (approximately) along the boresight, while strongly attenuating waves moving in other directions. There have been some speculations on the physical explanations for this behavior, including some suggested by this writer, but no one (to my knowledge) has yet set forth an analysis which is amenable to calculation.

It is the purpose of this paper to provide, in at least preliminary form, the theoretical analysis which is capable of explaining the spatial selectivity of the radar. The problem represents a fairly straightforward application of fourier transform theory. Preliminary calculations based on this theory demonstrate, for a representative case, that a high degree of 
spatial selectivity does, in fact, exist. There has been no attempt at this stage to obtain a detailed quantitative comparison of the predictions of this theory with experimental image data, however.

It appears from examination of images that this type of radar provides a potentially very powerful method for analyzing sea wave behavior and determining the directional properties of the sea wave spectrum. The analysis presented herein should provide some insight as to why this may be the case.

\section{The Derivation of the Radar Transfer Function}

A fixed (non scanning) beam real aperture radar (RAR) over looks the sea surface as illustrated schematically in Fig. 2 . The azimuth beamwidth is narrow and the radar is assumed to operate at LGA. The LGA assumption, typically about six degrees, permits us to ignore the variation of antenna gain in the elevation direction. The radar boresight is along $y$, the cross range direction is $x$, and $\bar{r}$ is a vector to a point in the $(x, y)$ plane. $\theta$ is the azimuth angle measured from the antenna boresight to the point $\bar{z}$. The illuminated patch, shown as the shaded area, is approximately. rectangular. The range extent of this patch is determined by the amount of data which is recorded and processed in the receiver. $R$ is the range to the center of the i.lluminated patch, and the patch size is taken to be small compared to $R$. The coordinate variable along the horizontal axis of the antenna aperture,: denoted by $u$, is taken to be parallel to. $x$. The (voltage) aperture illumination function of the antenna is denoted $A(u)$.

Shown within the illuminated patch is a single resolution cell of the radar. The angular extent of the cell is determined by the antenna azimuth beamwidth, while the range extent is determined by the signal bandwidth. The radars of interest have a resolution cell which is highly elongated (e.9., $1.5 \mathrm{~m}$ in $y$ and $44 \mathrm{~m}$ in $\mathrm{x}$ ). It is the shape of this cell which provides the preferential selectivity for surface waves whose phase fronts are approximately aligned with the long dimension of the cell, and thus are propagating approximately parallel to the radar boresight.

The geometry described here applies to the RSRE-Thorn EMI radar and the LLNL Hughes radar which were used to gather backscatter data in Loch Linnhe and the sound of sleat.

These radars produce images of backscatter intensity as a function of $y$ (or more correctly $r$ ) and $t$ ime $t$. These images are related to the normalized radar backscatter function, $\sigma_{0}(\bar{r}, t)$, through a radar transfer function which depends on the antenna gain pattern and the radar range resolution. We proceed to derive an expression for this transfer function both in terms of $x$ and $y$, and transform variables, $K_{x}$ and $K_{y}$. These functions will demonstrate the spatial selectivity of the radar.

The antenna voltage gain pattern $G(\theta)$ is related to the antenna aperture illumination $A(u)$ by 


$$
G(\theta) \sim \int A(u) e^{-i k u \sin \theta} d u
$$

where $k=2 \pi / \lambda$ is the wavenumber of the radar and a constant of proportionality has been ignored. For a small beamwidth and patch size (compared to $R$ ), we can approximate

$$
\sin \theta \cong \frac{x}{R}
$$

The antenna gain weighting function of $x$, denoted $G_{x}(x)$, is then given by the fourier transform relation

$$
G_{x}(x) \sim \int A(u) e^{-i(k x / R) u} d u
$$

The transform domain response, denoted $H_{x}\left(\mathbb{R}_{x}\right)$, is given by

$$
\begin{aligned}
H_{x}\left(K_{x}\right) & =\int e^{-i K_{x} x_{G_{x}}}(x) d x \\
& =2 \pi \int \delta\left(K_{x}+\frac{k u}{R}\right) A(u) d u \\
& =\frac{i \pi R}{k} A\left(\frac{K_{x} R}{k}\right)
\end{aligned}
$$

We have assumed here, without much loss of generality, that $A(u)$ is real and symmetric in $u$, from which it follows that both $G_{x}$ and $H_{x}$ are real and symmetric functions of their arguments.

The corresponding intensity weighting function of $x$ is denoted $I_{x}(x)$ and is a real symmetric function given by

$$
I_{x}(x)=G_{x}^{4}(x)
$$

The fourth power comes about because the voltage return contains the two-way antenna pattern and the result must be squared to obtain the intensity weighting. The intensity weighting in the $\mathbb{K}_{x}$ variable, denoted $J_{x}\left(\mathbb{K}_{x}\right)$, is obtained conveniently by noting that multiplication in the $x$ domain corresponds to convolution in the transform $\left(i . \theta,, \mathbb{R}_{x}\right)$ domain $[4] . J_{x}\left(R_{x}\right)$ is given by

$$
\begin{aligned}
J_{x}\left(K_{x}\right) & =\int e^{-i K_{x} x} I_{x}(x) d x \\
& \sim H_{x}\left(K_{x}\right) \otimes H_{x}\left(K_{x}\right) \otimes H_{x}\left(K_{x}\right) \otimes H_{x}\left(K_{x}\right) \\
& \sim A\left(K_{x} R / k\right) \otimes A\left(K_{x} R / k\right) \otimes A\left(K_{x} R / k\right) \otimes A\left(K_{x} R / k\right)
\end{aligned}
$$


where the convolution, denoted $\theta$, is over the $K_{x}$ variable and a proportionality constant has been ignored. $J_{x}\left(K_{x}\right)$ is also a real symmetric function.

Consider now the radar resolution in the range dimension. The (voltage) radar resolution function is given by the response of the matched filter in the receiver to a point target which produces a delayed replica of the transmitted signal. The matched filter, which provides a convenient means for performing correlation reception, produces at its output a signal which is the autocorrelation function, $\phi(\tau)$, of the transmitted signal. $\phi(\tau)$ is related to the signal voltage spectrum $S(f)$ by the transform relation

$$
\phi(\tau)=\int e^{-i 2 \pi f \tau}|S(f)|^{2} d f
$$

where $f$ is frequency. $S(f)$ is usually a narrowband function centered on the radar carrier frequency $f_{0}$. The output of the radar matched filter contains this phase which is removed by envelope detection when an intensity image is formed. Thus, we are justified in assuming that $S(f)$ is centered on zero frequency which has the effect of ignoring the carrier frequency. We shall also assume that $|S(f)|$ is symmetric about zero so that $\phi(\tau)$ is real and symmetric.

For a narrow beam RAR the time delay $\tau$ is

$$
\tau=\frac{2 r}{c} \cong \frac{2 y}{C}
$$

where $c$ is the velocity of light. The voltage response is a real symmetric function of $y$, denoted $G_{y}(y)$.

$$
G_{y}(y)=\int e^{-i 4 \pi f y / c}|S(f)|^{2} d f
$$

The response function in the transform variable $\mathbb{K}_{y}$, denoted $\mathrm{H}_{\mathrm{y}}\left(\mathrm{R}_{\mathrm{y}}\right)$, is a real symmetric function given by

$$
\begin{aligned}
H_{y}\left(K_{Y}\right) & =\int e^{-i K_{Y} G_{Y}}(y) d y \\
& =2 \pi \int \delta\left(K_{Y}+4 \pi f / c\right)|S(f)|^{2} d f \\
& =\frac{c}{2}\left|S\left(\frac{c K_{Y}}{4 \pi}\right)\right|^{2}
\end{aligned}
$$


The corresponding intensity weighting function is denoted $I_{Y}(Y)$ and is given by

$$
I_{Y}(Y)=G_{y}^{2}(Y)
$$

The intensity weighting function in the $\mathrm{K}_{\mathrm{y}}$ variable, denoted $J\left(R_{Y}\right)$, is once again conveniently obtained by noting that multiplication in the $y$ domain corresponds to convolution in the transform (i.e., $K_{y}$ ) domain.

$$
\begin{aligned}
J_{Y}\left(K_{Y}\right) & =\int e^{-i K_{Y} Y} I_{Y}(y) d y \\
& \sim H_{Y}\left(K_{Y}\right) \otimes H\left(K_{Y}\right) \\
& \sim\left|S\left(\frac{c K_{Y}}{4 \pi}\right)\right|^{2} \otimes\left|S\left(\frac{c K_{Y}}{4 \pi}\right)\right|^{2}
\end{aligned}
$$

This function is also real and symmetric in $\mathbb{R}_{\mathbf{y}}$.

The total intensity weighting function in $x$ and $y$, denoted $I(\bar{r})$, is given by the product

$$
I(\bar{y})=I_{x}(x) I_{y}(y)
$$

I here is basically a power resolution function for the radar, where $I_{x}$ and $I_{y}$ are given by (5) and (11). The corresponding intensity weighting function, denoted $J(\bar{K})$, is found to be a separable function of $\boldsymbol{K}_{\mathbf{X}}$ and $\boldsymbol{K}_{\mathbf{Y}}$.

$$
\begin{aligned}
J(\bar{K}) & =\iint e^{-i \bar{K} \cdot \bar{x}} I(\bar{r}) d \bar{x} \\
& =J_{X}\left(R_{X}\right) J_{Y}\left(R_{Y}\right)
\end{aligned}
$$

where $J_{x}$ is given by (6) and $J_{y}$ is given by $(12), \bar{k}=\left(K_{x}, K_{y}\right)$ is the surface wave propagation vector in $(x, y)$ space. $J(\bar{K})$ is seen to be a product of functions which are real and symmetric in $k_{x}$ and $\mathrm{K}_{\mathbf{y}}$.

To the extent that the normalized RCS, $\sigma_{0}(\bar{x})$, can be linearly related to the waves on the surface (a simplifying assumption which tends to become less valid as grazing angle is reduced), the function $J(\bar{K})$ can be viewed as the spatial wavenumber response of the RAR. It will be seen that $J(\bar{k})$ 
provides a highly selective response for waves which move in the radial direction. The role which $J(\bar{K})$ plays will become more apparent when we consider its effect on the space-time image, and the transform domain, $(\bar{R}, \Omega)$ space, image.

\section{A Representative Example}

We will now make specific assumptions on the form of the aperture illumination $A(U)$, the signal spectrum $S(f)$, and from these derive the form of the filter response function, $J(\bar{R})$, in wave number space. $J(\bar{K})$ will be normalized to unity at $K=0$, so constants of proportionality in the derivation will not matter.

The aperture illumination of the antenna, $A(u)$, is taken to be unity over an aperture width $D$ and zero outside this interval. Thus, $A(u)$ can be written

$$
A(u)=\operatorname{Rect}\left(\frac{u}{D}\right)
$$

From $(6), J_{x}\left(R_{x}\right)$ is given by

$$
J_{x}\left(K_{x}\right) \sim \operatorname{Rect}(\omega) \otimes \operatorname{Rect}(\omega) \otimes \operatorname{Rect}(\omega) \otimes \operatorname{Rect}(\omega)
$$

where

$$
\omega=\frac{R_{x} R}{k D}
$$

and the convolution is taken over the variable $\mathbf{R}_{\mathbf{x}}$. The order in which these convolutions are performed is immaterial. It can be shown, with some algebra, that the normalized $J_{x}\left(K_{x}\right)$ has the form

$$
\begin{array}{rlrl}
J_{x}\left(K_{x}\right) & =1-\frac{3}{2} \omega^{2}+\frac{3}{4}|\omega|^{3} & 0<|\omega|<1 \\
& =2-3|\omega|+\frac{3}{2} \omega^{2}-\frac{1}{4}|\omega|^{3} & 1<|\omega|<2 \\
& =0 & |\omega|>2
\end{array}
$$

The signal spectrum $S(f)$ is also taken to be rectangular, but with a bandwidth $B$ centered at zero frequency. Thus

$$
S(f)=\operatorname{Rect}\left(\frac{f}{B}\right)
$$

Note that

$$
|S(f)|^{2}=\operatorname{Rect}\left(\frac{f}{B}\right)
$$

From (12) and (20) it readily follows that 


$$
J_{y}\left(K_{y}\right) \sim \operatorname{Rect}(z) \otimes \operatorname{Rect}(z)
$$

where

$$
z=\frac{c x_{Y}}{4 \pi B}
$$

and the convolution is taken over $\mathbb{R}_{y}$. The normalized $J_{y}\left(\mathbb{R}_{y}\right)$ is readily shown to be

$$
\begin{array}{rlrl}
J_{Y}\left(K_{Y}\right)=1-|z| & 0<|z| & <1 \\
=0 & |z|>1
\end{array}
$$

For the purpose of this representative example, it is assumed that $D=50.8 \lambda$ and $B=100 \mathrm{MHz}$. These numbers correspond to a $3 \mathrm{~dB}$ one-way beamwidth of one degree and a range resolution of $1.5 \mathrm{~m}$. The assumptions approximate the parameters of the RSRE-Thorn EMI radar. The precise signal spectrum shape and antenna aperture illumination are, of course, not rectangular, but these assumptions will serve to demonstrate the spatial selectivity of interest.

Now we can compute $J(\bar{K})$ as the product of $J_{x}\left(R_{x}\right) J_{y}\left(R_{y}\right)$. We find it convenient, however, to express $J$ in terms of surface wavelength, $\Lambda$, and the angle $\alpha$ which $\bar{R}$ makes with the radar boresight along $y$. For this purpose we use the relations

$$
\begin{aligned}
& \mathrm{K}=2 \pi / \Delta \\
& \mathrm{R}_{\mathrm{x}}=\mathrm{R} \sin \alpha \\
& \mathrm{K}_{\mathrm{y}}=\mathrm{R} \cos \alpha
\end{aligned}
$$

The wave number response factor $J$ has been plotted vs. $\alpha$ for various values of surface wavelength in Fig. 3 . Since the curves are symmetric about $\alpha=0$, these have been plotted only for positive $\alpha$. When $\alpha=0$ the $J$ factor decreases with decreasing wavelength (increasing $K_{y}$ ) and drops to zero when the wavelength reaches 1.5 meters. This wavelength dependence is determined only by the spectrum of the signal. The J factor also decreases with increasing $\alpha$ (increasing $R_{x}$ ). The fall off is slow for long wavelengths, but is rapid for short wavelengths. [0f course, these short wavelengths would not be visible if the radar bandwidth was not large]. The observed wave groups in Loch Linnhe typically have a velocity of 1 meter per second, which corresponds to a surface wavelength of 2.56 meters. From the figure it is seen that surface wavelengths under three meters are very strongly attenuated, if not completely removed, for propagation angles greater than five degrees off the radar boresight.

Consider cross section modulations which are harmonically related to the wavelength of the underlying sinusoidal wave [5]. The effective wavelength which determines the spatial filter 
response will decrease much more rapidly with $\alpha$ when the effective wavelength is short, e.g., when $n$ is two or greater. When the waves have grown to the point of incipient or actual breaking and the curvature near the wave crest is high, the backscatter return-tends to be strongly concentrated near the wave crest. At LGA, shadowing of the wave trough also contributes to the spatial concentration of the backscatter RCS near the crest. The effect here is to redistribute the spatial backscatter energy from low to high wave numbers and thereby enhance the spatial selectivity for waves moving along the boresight.

For a typical Loch Linnhe image, of which Fig. 1 is an example, the range to the center of the illuminated patch is 2500 $\mathrm{m}$, the width of the patch is $44 \mathrm{~m}$ and the range extent of the patch is $384 \mathrm{~m}$ (256 pixels). A wave group starting at the center of one end of the patch and exiting a far corner would have (ignoring sign) $\alpha=3.28$ degrees, while a wave entering one corner of the patch and exiting the far corner would have an $\alpha=6.54$ degrees.

The predominant surface wavelengths in the field of view of the RAR depend strongly on environmental conditions such as wind speed, direction and fetch. For Loch Linnhe the fetch is quite I imited and the predominant surface wavelengths to be expected are substantially less, on the average, than one would expect in an open sea environment. The example image in Fig. 1 suggests that the principal group velocity is about $1.1 \mathrm{~m} / \mathrm{sec}$ and the corresponding predominant wavelength (along the range direction) is thus about three meters. Other images from this system, or radars similar to it, clearly show the presence of wave groups with various velocities, as one would expect. In some cases both inward and outward moving wave groups can be seen in the same image [3].

A cursory examination of the lifetime of these wave groups, which sometimes exceed 100 seconds, suggests that these are generally consistent with the very selective spatial filtering of moderate wavelength surface waves for this type of radar, as indicated in Table 1.

An additional, and potentially quite important, mechanism exists for enhancing radar returns from incipiently breaking waves. which are moving radially toward the radar. Scattering from a regular feature, e.g., a wedge-like shape, has a backscatter RCS which, like a metal rod, exhibits a specular return when the wave crest is perpendicular to the LOS. This type of specular does not require some portion of the sea surface to be normal to the LOS. It is necessary that shape near the wave crest (regardless of whether the wave is breaking) be approximately maintained as one moves along the crest. The enhancement which this effect produces for radially moving waves has a complex dependence on the statistical behavior of shapes near the crests and no attempt is made here to model and quantify this effect. However, the spatial selectivity of this scattering behavior should be superimposed on that which has been calculated above. 
Although the above analysis has assumed LGA operation, it should be noted that a high degree of spatial selectivity of surface waves can be similarly obtained with radars which operate at intermediate grazing angles, and that the analysis presented herein can be readily extended to this regime.

4. The Image Response Function in Transform Space

Consider the normalized RCS function $\sigma_{0}(\bar{r}, t)$ which is a function of space and time. Let $\Sigma_{\circ}(\bar{K}, \Omega)$ be the Fourier transform of $\sigma_{0}(\bar{r}, t)$ given by

$$
\Sigma_{0}(\bar{R}, \Omega)=\iiint e^{-t(\bar{K} \cdot \bar{r}-\Omega t)} \sigma_{0}(\bar{r}, t) d \bar{r} d t
$$

where $\bar{K}$ as previously is the wave number vector and $\Omega$ is the radian frequency. Suppose we identify each Fourier component of oo with a surface wave which moves with the usual deep water dispersion relationship.

$$
\Omega=\Omega(K)=\sqrt{g K}
$$

Then $\Sigma_{0}$ would have the form

$$
\Sigma_{\circ}(\overline{\mathrm{K}}, \Omega)=\pi\left[\mathrm{W}(\overline{\mathrm{K}}) \delta(\Omega-\Omega(\mathrm{K}))+\mathrm{W}^{*}(-\overline{\mathrm{K}}) \delta(\Omega+\Omega(\mathrm{K}))\right]
$$

where $W(\bar{R})$ characterizes the directional properties of the surface wave spectrum. This form for $\Sigma_{0}$ assures that $\sigma_{0}$ will be real. The voltage spectrum for surface waves, $P(\bar{R})$, is found from (27) by integrating over $\boldsymbol{\Omega}$.

$$
P(\bar{K})=\frac{1}{2 \pi} \int \Sigma_{0}(\bar{K}, \Omega) d \Omega=\frac{1}{2}\left[W(\bar{K})+W^{*}(-\bar{K})\right]
$$

which is a conjugate symmetric function of $\overline{\mathbf{K}}$. Now we proceed to examine the form of the image intensity function; denoted $U(y, t)$, which is given by

$$
U(y, t)=\iint I(x, y-v) \sigma_{0}^{\vdots}(x, v, t) d v d x
$$

where I, as noted earlier, plays the role of a power resolution function. The integration over $x$ simply represents the power weighting of the antenna beam pattern. The integration over the dummy variable $v$ corresponds to a convolution in range of the resolution function with $\sigma_{0}$. An implicit assumption in (29) is that the radar return becomes rapidly decorrelated (i.e., due to random phase) with changes in $x$ or $y[6]$. Thus, any weighted sum 
or integration over the image can be characterized as a power addition.

The Fourier transform of $U(Y, t)$. denoted $Z\left(R_{y}, \Omega\right)$, can be found from (29) with the aid of (14) and (25). The derivation involves a straightforward application of Fourier transform methods, but the details are omitted here. The result is

$$
\begin{aligned}
Z\left(K_{Y}, \Omega\right) & =\iint e^{-i\left(K_{Y} y-\Omega t\right)} U(y, t) d y d t \\
& =\frac{1}{2 \pi} \int J\left(K_{X}, K_{y}\right) \Sigma_{o}\left(K_{X}, K_{Y}, \Omega\right) d K_{X}
\end{aligned}
$$

It is clear now from this expression that the wave number response factor $J$ operates directly on the wave number components of $\sigma_{0}$.

It is of interest to derive an expression for $Z\left(K_{y}, \Omega\right)$ so that we can examine its properties. Insert (27) for $\Sigma_{0}$ in the above expression for $\mathrm{z}\left(\mathrm{R}_{\mathbf{y}}, \boldsymbol{\Omega}\right)$.

$$
\mathrm{Z}\left(\mathrm{K}_{\mathrm{y}}, \Omega\right)=\frac{1}{2} \int J(\overline{\mathrm{K}})\left[\mathrm{W}(\overline{\mathrm{K}}) \delta(\Omega-\Omega(\mathrm{K}))+\mathrm{W}^{*}(\overline{-\bar{K}}) \delta(\Omega+\Omega(\mathrm{K}))\right] d \mathrm{~K}_{\mathrm{x}}
$$

We use the gravity wave dispersion relation of (26). When $\Omega>0$ only the first term contributes to the integral. The value of $k_{x}$ which satisfies $(26)$ is denoted $R_{x}^{\circ}$ and is given by

$$
K_{x}^{\circ}=\left[\Omega^{4} g^{-2}-K_{y}^{2}\right]^{\frac{2}{2}}
$$

Note that $-\mathbb{K}_{x}^{\circ}$ also satisfies the dispersion relationship. When $\boldsymbol{\Omega}<0$ only the second term contributes to the integral. These contributions once again occur where $\mathbf{K}_{x}= \pm \mathbb{R}_{x}^{\circ}$. relation

The integral over a $\delta$-function can be evaluated using the

$$
\int G(v) \delta(F(v)) d v=G\left(v_{0}\right)\left|\frac{\partial g}{\partial v}\right|_{v_{0}}^{-1}
$$

where $v_{0}$ is the solution of

$$
F(v)=0
$$

In our case there are two solutions for $v_{0}$ which contribute to the integral.

One can readily show

$$
\left|\frac{\partial}{\partial K_{x}}(\Omega \pm \Omega(K))\right|_{ \pm K_{x}^{\circ}}=\frac{1}{2} g^{2} \Omega^{-3} K_{x}^{\circ}
$$


Then, recalling that $J\left(K_{x}, K_{y}\right)$ is symmetric in $K_{x}$, we have for $\mathrm{Z}\left(\mathrm{K}_{\mathrm{x}}, \Omega\right)$

$$
\begin{array}{ll}
Z\left(K_{y}, \Omega\right)=g^{-2} \Omega^{3} K_{x}^{\circ-1} J\left(K_{x}^{\circ}, K_{y}\right)\left[W\left(K_{x}^{\circ}, K_{y}\right)+W^{*}\left(-K_{x}^{\circ}, K_{y}\right)\right], \quad \Omega>0 \\
Z\left(K_{y}, \Omega\right)=g^{-2} \Omega^{3} K_{x}^{\circ-1} J\left(K_{x}^{\circ}, K_{y}\right)\left[W\left(-K_{x}^{\circ},-K_{y}\right)+W^{*}\left(K_{x}^{\circ},-K_{y}\right)\right], \quad \Omega<0
\end{array}
$$

The presence of $\Omega^{3}$ tends to emphasize the higher frequencies and wavenumbers. $Z\left(K_{y}, \Omega\right)$ has a singularity which gives a large value where

$$
\mathbf{K}_{\mathbf{x}}^{0} \equiv \mathbf{0}
$$

which is along the dispersion curve given by

$$
\Omega=\left[g K_{\mathrm{y}}\right]^{\frac{1}{2}}
$$

This dispersion curve has been clearly observed in computed ( $R, \Omega$ ) images for LGA RAR data (see, for example, [1] ).

Consider the extension of the above analysis to the case where each component surface wave, with wavelength $\Lambda$ say, manifests itself in the RCS image not only as a wavelength $\Lambda$, but also as a series of harmonics with wavelength $\Lambda / n$. If $K$ is the observed wave number for the nth harmonic, the correct wave number for the underlying surface wave is $\mathrm{K} / \mathrm{n}$, and the dispersion relation for the wave is

$$
\Omega=\sqrt{g \hat{K} / \Omega}
$$

The above analysis which has been given for the fundamental wave, $n=1$, applies directly for all harmonics of these waves, the only change being that one must use the above dispersion relation for each of the harmonics. 


\title{
GLOSSARY OF SYMBOLS AND ACRONYMS
}

\author{
$A(u)=$ antenna illumination pattern \\ $B=$ bandwidth of transmitted signal \\ $c=$ velocity of light \\ $D=$ width of antenna \\ $f=$ radar frequency \\ $f_{0}=$ radar carrier frequency \\ $\mathbf{G}_{\mathbf{x}}=$ voltage resolution function in $\mathbf{x}$ \\ $G_{y}=$ voltage resolution function in $y$ \\ $\mathrm{H}_{\mathbf{x}}=$ voltage resolution function in $\mathrm{K}_{\mathbf{x}}$ \\ $H_{y}=$ voltage resolution function in $K_{y}$ \\ $I_{x}=$ intensity weighting function in $x$ \\ $I_{y}=$ intensity weighting function in $Y$ \\ $I(x, y)=$ image weighting function \\ $J_{x}=$ intensity weighting function in $k_{x}$ \\ $J_{y_{-}}=$intensity weighting function in $R_{y}$ \\ $J(\bar{R})=$ filter response function in transform space \\ $\overrightarrow{\mathrm{K}}=\left(\mathrm{K}_{\mathrm{x}}, \mathrm{K}_{\mathrm{y}}\right)=$ surface wave propagation vector \\ $k=2 \pi / \lambda$ \\ $\mathrm{n}=$ harmonic number of wave \\ $P(\bar{K})=$ voltage spectrum of surface waves \\ $\bar{y}=(x, y)$ \\ $\mathrm{R}=$ average range to radar patch \\ $S(f)=$ voltage spectrium of transmitted signal \\ $t=$ time \\ $\tau=$ time delay after transmit pulse \\ $U(y, t)=$ radar output intensity function \\ $\mathrm{u}=$ antenna coordinate variable \\ $W(\bar{R})=$ voltage spectrum of RCS image \\ $x=$ cross range (down track) coordinate \\ $y=$ range coordinate \\ $v, w, z=$ dummy variables \\ $\mathrm{Z}\left(\mathrm{K}_{\mathrm{y}}, \Omega\right)=$ image intensity in $\left(\mathrm{R}_{\mathrm{y}}, \Omega\right)$ coordinates \\ $\alpha=$ wave direction off boresight \\ $\boldsymbol{\Omega}=$ surface wave radian frequency \\ $\Lambda=$ surface wavelength \\ $\lambda=$ radar wavelength \\ $\sigma_{0}(x, y, t)=$ normalized RCS vs. position and time \\ $\Sigma_{0}\left(R_{x}, R_{y}, \Omega\right)=$ normalized $R C S$ in $\left(R_{x}, R_{y}, \Omega\right)$ coordinates \\ $\theta=$ angle off antenna boresight \\ $\phi(\tau)=$ autocorrelation function of time waveform \\ IINL=Lawrence Livermore National Laboratory \\ LGA = low grazing angle \\ RAR = real aperture radar \\ LOS = line of sight \\ RCS=radar cross section \\ RSRE=Royal Signals and Radar Establishment
}




\section{REFERENCES AND COMMENTS}

1. Ben 0. Werle, "Sea Backscatter, Spikes and Wave Group Observations at Low Grazing Angles," planned for publication in IEEE "Radar "95". Prepublication copy courtesy Swerling, Manasse and Smith(SMS), Inc. 6/27/94.

2. Personal communication, Marshall Tulin, UCSB. A paper on this subject is being prepared for publication.

3. Personal communication, LLNL and SMS personnel.

4. See, for example, A. Papoulis, "The Fourier Integral and Its Applications," (McGraw Hill, Inc., 1962), p. 26-27.

5. The radar cross section, $\sigma_{0}(\vec{r}, t)$, is a nonlinear functional of surface height both because of nonlinear hydrodynamics and because wave height manifests itself in the scattering integrals in a nonlinear manner. Not only will an isolated sinusoidal wave produce harmonics in the image, but different waves will interact to produce wave beats (analogous to the mixing of different frequencies in a nonlinear receiver) which will appear in the image.

6. D. Holliday, G. St-Cỳr, N. E. Vloods, "A Radar Ocean Imaging Model for Small to Moderate Incidence Angles," Int. J. Remote Sensing, 1986, Vol. 7, No. 12, 1809-1834. 


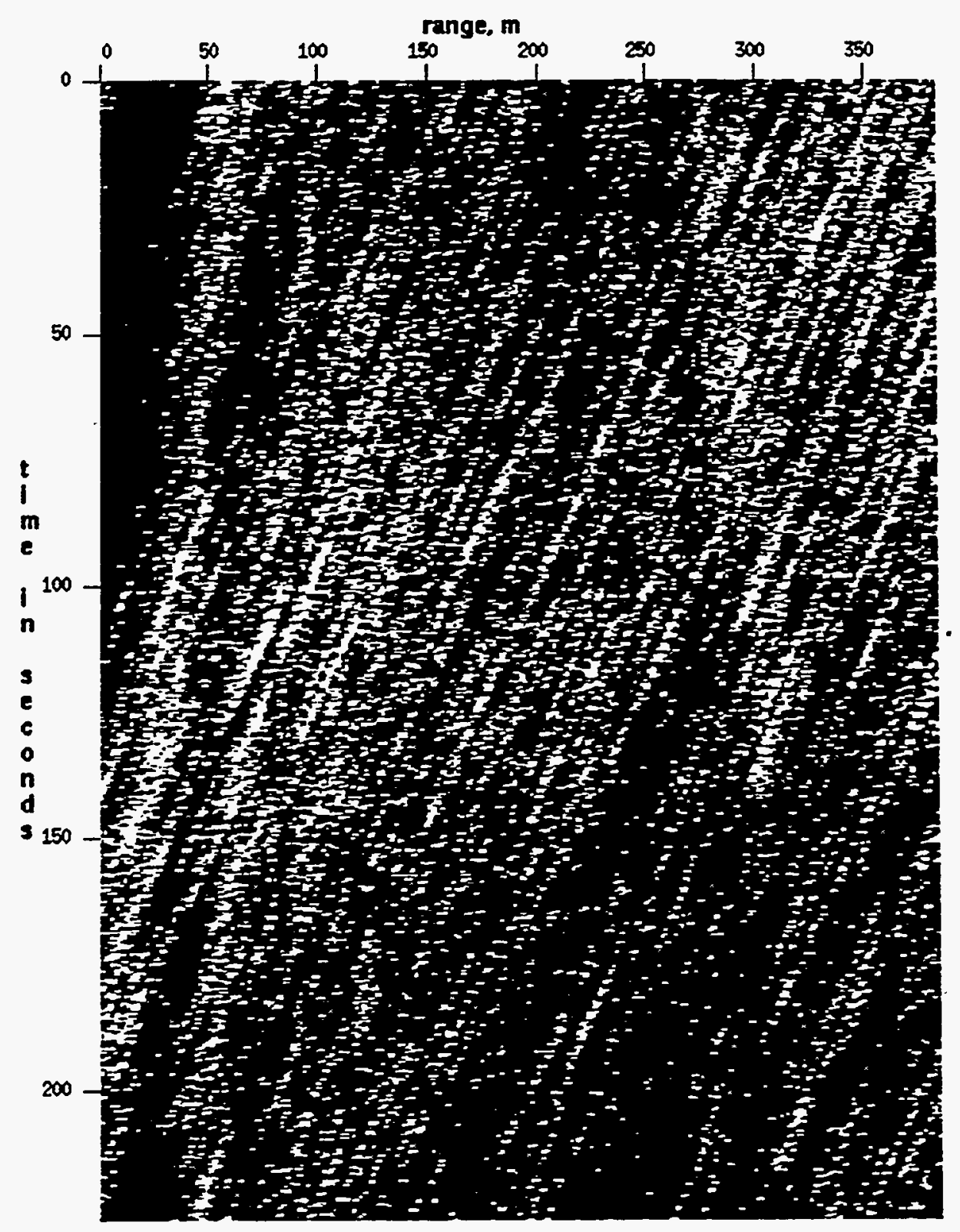

Figure 1. HH Backscatter for LI90 Run 16, 16:23:15 to 16:27:01 GMT, 64 Pulse Averaging ( 0.128 Seconds) 


\section{FIG 2. SYSTEX GEOMIEILYY \\ (TOP VIEW--NOT TO SCALE)}

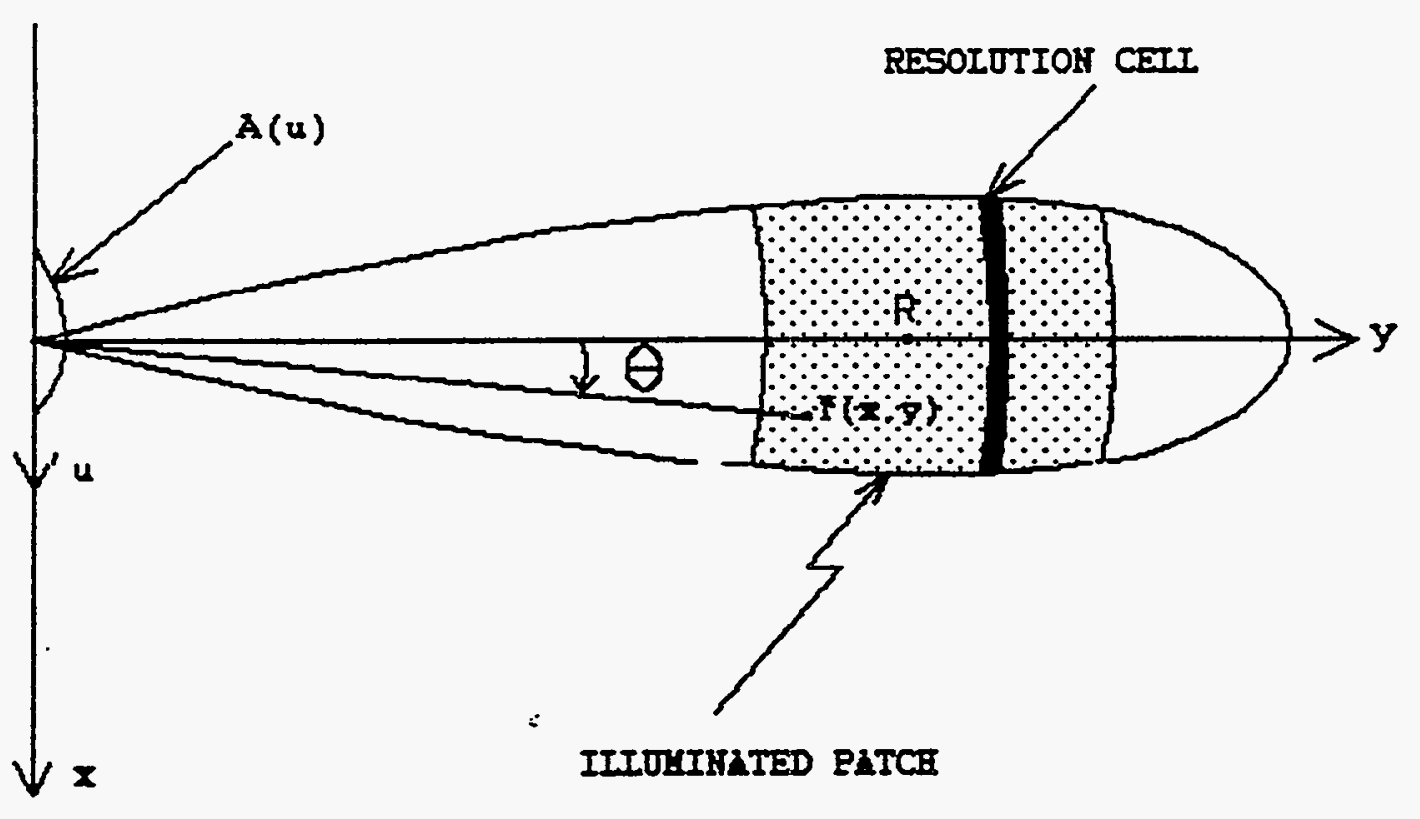




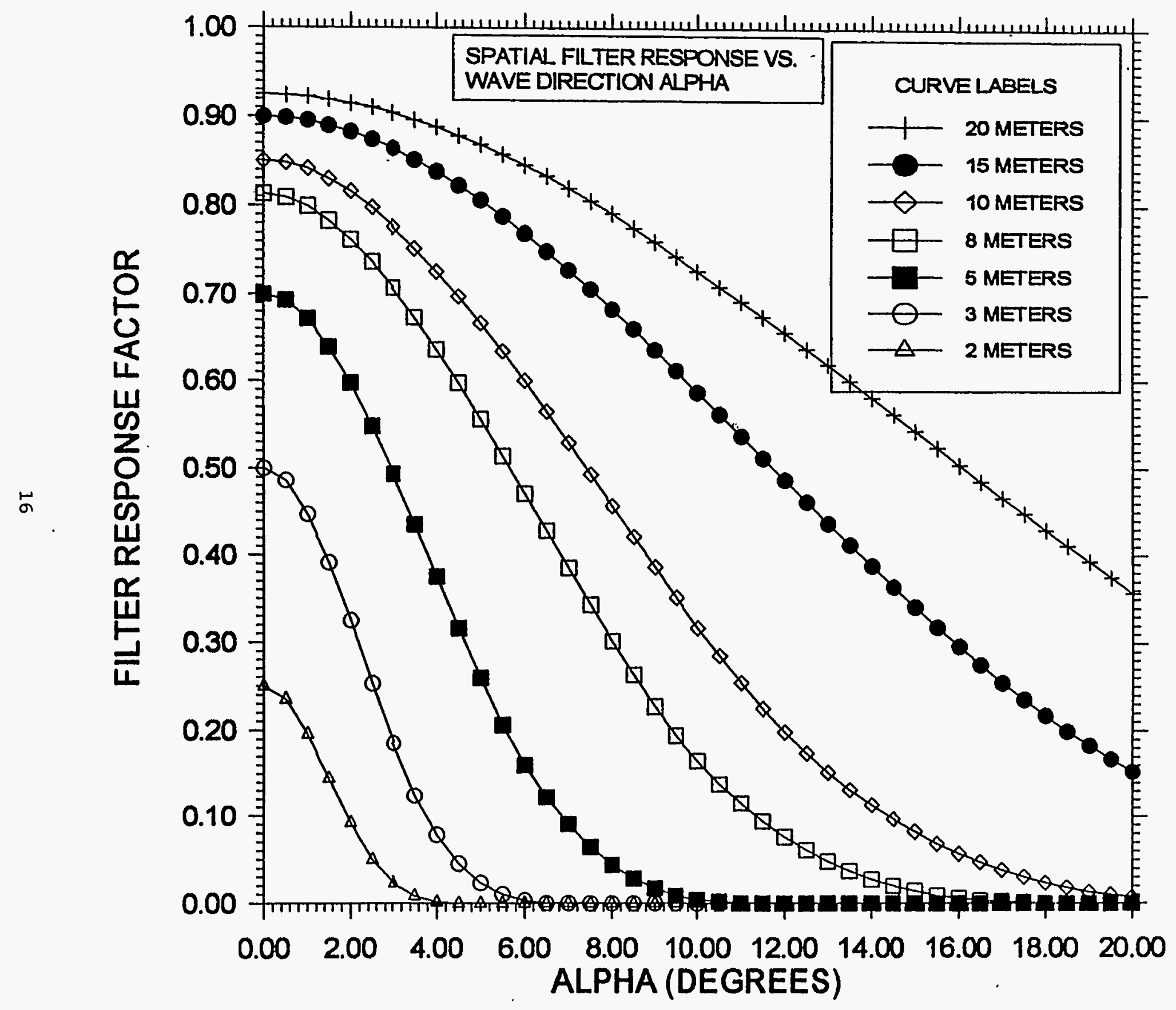

Figure 3 